\title{
Using Queue Statistics in Beamforming for ALOHA
}

\author{
Vidyut Naware and Lang Tong \\ School of Electrical and Computer Engineering \\ Cornell University, Ithaca, NY 14853. \\ Email :- \{vidyut, ltong $\} @ e c e . c o r n e l l . e d u$
}

\begin{abstract}
We address the problem of receiver design for the uplink of a WLAN network from a stability viewpoint. We propose a cross layer approach that incorporates the users' buffer statistics for the design of smart antennas employed by the base station/access point. In particular, we consider slotted ALOHA as the MAC protocol and we propose a linear MMSE front-end that utilizes the probability of a users' queue being empty in its design. We compare the performance of the proposed MMSE front-end with the best adaptive front-end for the two user case and show that under certain conditions the proposed front-end is close to optimal.
\end{abstract}

\section{INTRODUCTION}

The traditional layered network architecture uses a modular approach in the design of the various layers; all the layers are designed separately so as to simplify network design. A recent line of work suggests that it is worthwhile to consider the intrinsic "coupling" that exists between the different layers [1]-[3] and to follow the so called "cross-layered" approach for network design. This involves a joint design of two or more layers so as to gain maximum benefit from the layers involved.

Traditional approaches to the design of PHY and MAC layers were based on abstractions like the collision channel viz., the MAC layer was designed to avoid "collisions" that resulted from simultaneous transmissions from more than one user. The PHY layer was assumed to be incapable of handling multi-user interference. Similarly, the PHY layer was designed assuming users always have data to send and thus neglected the issue of source burstiness. However, advanced receiver design techniques have mitigated effects of multi-user interference whereas the increasing demand for data traffic over networks has brought the issue of source burstiness to the fore. Thus, PHY and MAC layer designs based on abstractions and assumptions like the collision channel are rendered invalid in modern wireless communication systems. Hence, there

This work was supported in part by the National Science Foundation under Contract CCR-0311055, the Multidisciplinary University Research Initiative under the Office of Naval Research Contract N00014-00-10564, and by the ARL CTA on Communications and Networks under Grant Contract DAAD19-01-2-011. is a need to analyze alternative cross-layer design strategies based on better modelling of interaction amongst layers.

In this paper, we take a cross-layer approach and look at the problem of multiple antenna receiver design for the uplink of a WLAN system that uses slotted ALOHA as the random access mechanism. Recently, it has been shown [3] that in a certain regime of spatial diversity, slotted ALOHA is the optimal MAC protocol and this is why we choose ALOHA as the MAC in our setup. The objective is to design the PHY layer using information available from the MAC (ALOHA) layer. The information in this case is the probability that a user has a packet available in his queue to transmit. The criterion that we use for this joint design is the stability of the users' queues.

The remainder of the paper is organized as follows. In Section II, we provide the general problem setup and review some concepts and results on stability of the ALOHA protocol with multipacket reception. In Section III, the linear MMSE receiver based on queue statistics is discussed and a comparison of the proposed receiver with other standard receivers is provided. We conclude with Section IV.

\section{SYSTEM MODEL AND BACKGROUND}

We consider a wireless LAN of $N$ users communicating with an access point. The access point may have multiple antennas for beamforming or for other diversity techniques, thus allowing simultaneous transmissions to be received. The $i$ th user generates packets at the rate of $\lambda_{i}$. Each user has a buffer for arriving and backlogged packets. The channel is slotted, and the slot duration equals to the packet transmission time. The state of the queue in slot $t$ is defined by the number of packets $Q_{i}^{t}$ in the queue at the beginning of the slot.

For a system involving a set of $N$ users $\mathcal{U}=$ $\{1,2, \ldots, N\}$, we consider a multiuser physical layer defined by a set of conditional probabilities. For any subset of users $\mathcal{T} \subseteq \mathcal{U}$ transmitting in a slot, the probability of 


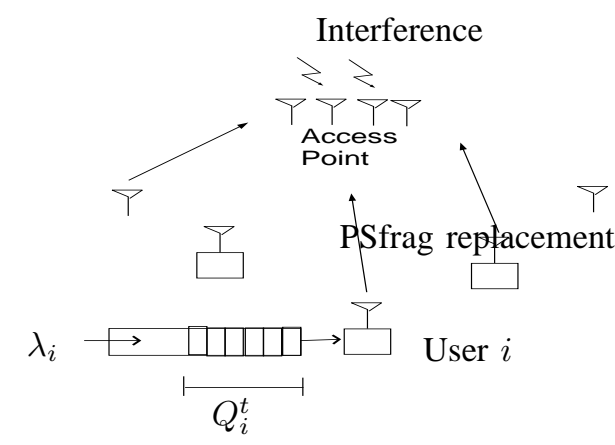

Fig. 1. The general multiple-access setup with queues.

successfully receiving packets from $\mathcal{R} \subseteq \mathcal{T}$ is given by

$P_{\mathcal{R}, \mathcal{T}}=\operatorname{Pr}\{$ only packets from users in $\mathcal{R}$ are successfully received| users in $\mathcal{T}$ transmit\}.

The set of conditional probabilities $\mathcal{P}=\left\{P_{\mathcal{R}, \mathcal{T}}, \mathcal{T} \subseteq\right.$ $\left.2^{\mathcal{U}}, \mathcal{R} \subseteq \mathcal{T}\right\}$ completely specifies the probability space for reception. Note also that all physical layer characteristics are summarized by $\mathcal{P}$ in the sense that, for each PHY configuration, there is a corresponding probability space $\mathcal{P}$.

A primary concern in MAC design is stability. By stability* we mean that the probability of buffer overflow can be made arbitrarily small by making the buffer size sufficiently large [4]-[7]. The stability region is a set $\mathcal{S}$ of (arrival) rate vectors such that for each rate vector $\boldsymbol{\lambda}=\left[\lambda_{1}, \cdots, \lambda_{N}\right] \in \mathcal{S}$, there exists a MAC protocol that makes all queues stable. The stability region for a particular MAC protocol is the set of rates that are stable under that MAC. For example, the stability region of ALOHA $\mathcal{S}_{\text {ALOHA }}$ is the set of all arrival rate vectors that are stable under ALOHA. By definition, $\mathcal{S}_{\text {ALона }} \subseteq \mathcal{S}$. It turns out that the achievable rates and the stability regions depend only on a set of marginal conditional probabilities

$$
P_{i \mid \mathcal{T}} \triangleq \sum_{\mathcal{R}: i \in \mathcal{R}} P_{\mathcal{R}, \mathcal{T}}
$$

where $P_{i \mid \mathcal{T}}$ is the probability that the $i$ th user is successfully received given users in $\mathcal{T}$ transmit.

The ALOHA protocol can be parameterized by a vector of transmission probabilities $\mathbf{p}=\left[p_{1}, \cdots, p_{N}\right]$. Node $i$ transmits a packet with probability $p_{i}$ if its queue is not empty. If $\boldsymbol{\lambda} \in \mathcal{S}_{\mathrm{ALOHA}}$, then there exists a $\mathbf{p}$ such that all queues are stable. The ALOHA stability region for an $N$ user system is unknown in general. For the collision channel, characterizing stability region has been a long

${ }^{*}$ Let $\mathbf{Q}^{t}=\left(Q_{1}^{t}, \ldots, Q_{N}^{t}\right)$ be the queue lengths of an $N$ user system. The system is stable if for $\mathbf{x} \in \mathbb{N}^{N}, \lim _{t \rightarrow \infty} \operatorname{Pr}\left\{\mathbf{Q}^{t}<\mathbf{x}\right\}=$ $F(\mathbf{x})$ and $\lim _{\mathbf{x} \rightarrow \infty} F(\mathbf{x})=1$. standing open problem. There is, however, a simple case when an antenna array is used. If a zero-forcing antenna array is used to eliminate all interfering nodes, we then have $N$ independent MAC channels, albeit each may have a much smaller stability region due to noise enhancement of the zero-forcing operation.

Recently, the stability region of ALOHA for the two user case has been characterized and it has been shown that in a certain regime of multipacket reception capability, ALOHA is optimal in the sense that ALOHA can stabilize all stable arrival rates [3], [8]. Specifically, in the two user case it has been shown that $\mathcal{S}_{\text {ALOHA }}=\mathcal{S}$ provided that $P_{1 \mid 1} \geq P_{1 \mid(1,2)}, P_{2 \mid 2} \geq P_{2 \mid(1,2)}$ and

$$
P_{2 \mid(1,2)} \geq\left(P_{1 \mid 1}-P_{1 \mid(1,2)}\right) \frac{P_{2 \mid 2}}{P_{1 \mid 1}} .
$$

Further, once ALOHA is optimal no transmission control is required i.e., ALOHA with transmission probability one is optimal. This result has the following implication-if the PHY layer behavior is modelled accurately, an efficient and simple MAC layer can be designed. This result makes a case for cross-layer design in which the MAC layer can be tuned according to PHY layer capabilities.

\section{Using QUEUE STATISTICS IN BEAMFORMING}

In this work, we explore cross-layer design in the reverse direction viz., we analyze performance of a PHY layer that explicitly uses MAC layer information in its design. We begin by specifying the channel propagation model. The receiver provides multipacket reception capability by employing a linear array of $M$ antennas. We assume that users are located relatively far away from the base station at fixed angular postions and that most of the energy from user transmissions is received from a planar wavefront arriving at that angle $\boldsymbol{\theta}$. Under these assumptions, we can describe the received signal at the base station $\mathbf{y}(t)$ at time $t$ as

$$
\mathbf{y}(t)=\mathbf{V H}(t) \mathbf{s}(t)+\mathbf{n}(t)
$$

where $\mathbf{V}$ is a matrix of array responses, $\mathbf{H}(t)=\operatorname{diag}\left[h_{1}(t), h_{2}(t), \cdots, h_{N}(t)\right]$, a diagonal matrix of channel (flat) fading for the users, $\mathbf{s}(t)=\left[s_{1}(t), s_{2}(t), \cdots, s_{N}(t)\right]^{T}$, a vector of users' transmitted symbols and $\mathbf{n} \sim \mathcal{C N}\left(0, \mathbf{I}_{M}\right)$, additive white gaussian noise.

We also assume that the channel fading is slow and it is independant for the users and also is i.i.d. from slot to slot. For our results, we assume Rayleigh fading with zero mean and covariance matrix $\Sigma=$ $\operatorname{diag}\left[\sigma_{h_{1}}^{2}, \sigma_{h_{2}}^{2}, \cdots, \sigma_{h_{N}}^{2}\right]$. User symbols $\left(s_{i}\right)$ are independant of each other and the channel fading with $\left|s_{i}\right|^{2}=$ $1, i=1,2, \cdots, N$. We represent the front-end processing 
by $\mathbf{F}^{\mathbf{H}}(t)$ ( $i$ th row of $\mathbf{F}^{\mathbf{H}}(t)$ is the set of beamforming weights for the $i$ th user) as follows:

$$
\begin{aligned}
\hat{\mathbf{s}}(t)=\mathbf{F}^{\mathbf{H}}(t) \mathbf{y}(t) & =\overbrace{\mathbf{F}^{\mathbf{H}}(t) \mathbf{V H}(t)}^{\mathbf{R}(t)} \mathbf{s}(t)+\mathbf{F}(t) \mathbf{n}(t) \\
& =\mathbf{R}(t) \mathbf{s}(t)+\mathbf{w}(t)
\end{aligned}
$$

We assume a SINR threshold model for packet success i.e., a packet is successfully received and decoded for user $i$ (with SINR $\gamma_{i}(\mathbf{H})$ ) if

$$
\gamma_{i}(\mathbf{H}) \triangleq \mathbb{E}\left(\operatorname{SINR}_{i} \mid \mathbf{H}\right)>\beta_{i},
$$

where $\beta_{i}$ is a threshold determined by the Quality of Service (QoS) requirement of the users.

Note that under the SINR threshold model, there is a possibility of receiving more that one packet successfully at the receiver. Given a beamformer $\mathbf{F}$, the probability space for packet reception as defined in the previous section $\mathcal{P} \mathbf{F}$ is given by

$$
P_{i \mid \mathcal{T}}^{\mathbf{F}}=\operatorname{Pr}\left\{\gamma_{i}(\mathbf{H})>\beta_{i} \mid \mathcal{T} \text { transmits }\right\} .
$$

Thus, each beamformer corresponds to a different reception model and hence a different ALOHA stability region $\mathcal{S}_{\mathbf{F}}$. For a class $\mathcal{F}$ of beamformers, we define the stability region as

$$
\mathcal{S}=\bigcup_{\mathbf{F} \in \mathcal{F}} \mathcal{S}_{\mathbf{F}} .
$$

We can now examine a few beamforming strategies and compare them in terms of their stability region. These strategies could be coherent or non-coherent depending on whether the channel $\mathbf{H}$ is known at the receiver. We assume that the deterministic and time-invariant array response $\mathbf{V}$ is known at the receiver.

Traditional design approaches analyze the performance of the following front-ends.

1) Matched Filter (MF): This is the simplest form of beamforming with $\mathbf{F}=\mathbf{V}$. It is non-coherent and assumes no knowledge of the queue states of the users. It is not optimal (in terms of maximizing SNR) unless only one user transmits.

2) Pseudo-MMSE (pMMSE): If the receiver assumes that all users have packets to be transmitted, then a non-coherent MMSE beamformer can be implemented with $\mathbf{F}^{\mathbf{H}}=\operatorname{diag}\left[\sigma_{h_{1}}^{2}, \sigma_{h_{2}}^{2}, \cdots, \sigma_{h_{N}}^{2}\right] \mathbf{V}^{H}(\boldsymbol{\theta}) \mathbf{R}_{y y}^{-1}$, where $\mathbf{R}_{y y}$ is the correlation matrix of $\mathbf{y}$ assuming all users transmit.

We also consider the performance of a coherent "clairvoyant" MMSE beamformer that assumes complete knowledge of which users transmit in a slot. This receiver is optimal in the sense that it maximizes the SINR of every user. This receiver provides an outer bound to the stability region for any class $\mathcal{F}$ of receivers but is impractical as it assumes perfect knowledge of channel states. Further, this receiver has a training overhead to detect exactly which users are transmitting in a slot and estimate the channel.

\section{A. Proposed Receiver}

We propose a non-coherent MMSE front-end which incorporates the stationary probability that a user has a non-empty queue in the above system provided that the queues are stable. In the queueing system described above (which is a Markov Chain), stability is equivalent to the existence of a stationary (or limiting) distribution of the queue evolution process and so the stationary probability that a user has a non-empty queue exists. Let $\mathbf{z}=\mathbf{H s}$. Our MMSE receiver has the following form.

$$
\mathbf{F}_{\mathbf{m m s e}}^{\mathbf{H}}=\mathbf{R}_{\mathbf{z z}} \mathbf{V}^{\mathbf{H}}\left(\mathbf{V R}_{\mathbf{z z}} \mathbf{V}^{\mathbf{H}}+\sigma^{2} \mathbf{I}\right)^{-1}
$$

where

$$
\begin{aligned}
\mathbf{R}_{\mathbf{z z}} & =\mathbb{E}\left(\mathbf{z z}^{\mathbf{H}}\right) \\
& =\operatorname{diag}\left(\sigma_{h_{1}}^{2} \mathbb{E}\left|s_{1}\right|^{2}, \cdots, \sigma_{h_{N}}^{2} \mathbb{E}\left|s_{N}\right|^{2}\right)
\end{aligned}
$$

In the above equation, the expectation is over the random user symbols, the stationary probability that a user has atleast a packet in his queue and the ALOHA retransmission probability. Now, the stationary probability that a user has atleast a packet in his queue depends on the actual front-end which is used at the receiver. Note that since the channel realization and user symbols are independant, we only need the marginal stationary probabilities of each individual queue being non-empty in order to compute $\mathbf{R}_{\mathbf{z z}}$. Define $\rho_{i}=\mathbb{E}\left|s_{i}\right|^{2}$. Then, since $\left|s_{i}\right|^{2}=1$, we have

$$
\rho_{i}=p_{i} \operatorname{Pr}\{\text { Queue } i \text { is non-empty }\}
$$

If we could compute $\rho_{i}$, then we could implement the proposed MMSE receiver. Unfortunately, there are no known results on the stationary probabilities of the queues in an ALOHA system even for the two user case (except for the collision channel) and it seems like an intractable problem [9]. So, we parametrize the class of MMSE receivers just described by $\boldsymbol{\rho}=\left[\rho_{1}, \cdots, \rho_{N}\right] \in[0,1]^{N}$ and find the stability region for this class of receivers numerically.

\section{B. Results}

To compare the performance of various front-ends with the proposed MMSE receiver, we consider the two user case. The primary reason for looking at the two user case is that the stability region of ALOHA for the two user case for general packet reception models is known. 


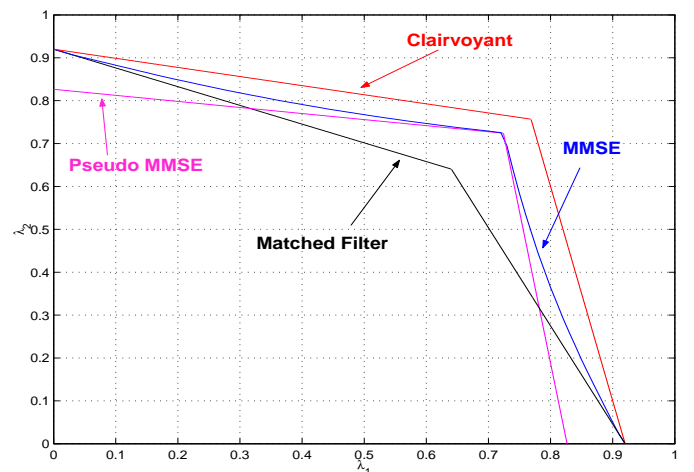

Fig. 2. Stability region of beamformers with equal power users. $M=3$, $\boldsymbol{\theta}=[54,72], \beta_{1}=\beta_{2}=-3 d B, \sigma_{h_{1}}^{2}=\sigma_{h_{2}}^{2}=3 d B$.

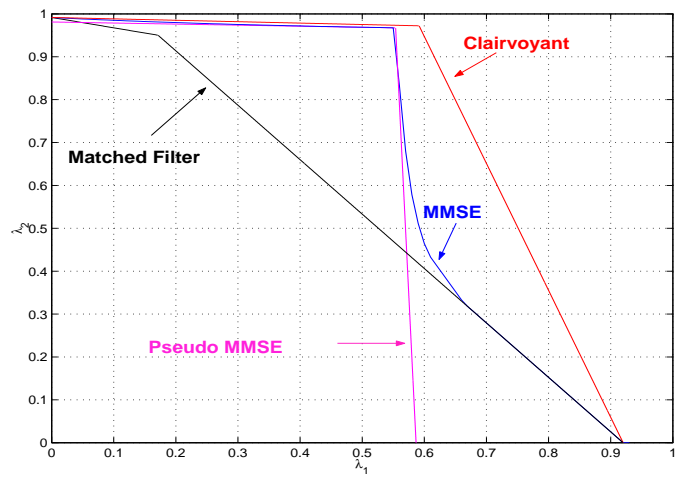

Fig. 3. Stability region of beamformers with unequal power users (nearfar effects). $M=3, \boldsymbol{\theta}=[54,72], \beta_{1}=\beta_{2}=-3 d B, \sigma_{h_{1}}^{2}=3 d B$, $\sigma_{h_{2}}^{2}=13 d B$.

Figures 2 and 3 show the stability region of different beamformers and illustrate performance of the beamformers in two cases of interest. Figure 2 is the case in which the two users have equal power with well conditioned channels. In this case, the simple nonadaptive matched filter receiver offers nearly as large a stability region as the clairvoyant beamformer. This indicates that the gain of using sophisticated signal processing may not justify the complexity of implementations. Figure 3 is the case when one user has significantly stronger power then the other. The difference among these receivers becomes evident. The simple matched filter offers a substantially smaller stability region than that of the clairvoyant beamformer. However, if both users operate in the lower left corner of the rate region, the matched filter beamformer will perform reasonably well because it is often the case that only one user has packets to transmit. The pseudo-MMSE that assumes both users always have packets to transmit can perform poorly in the high rate region of the weaker user. In fact, despite its more complex implementation, the pseudo-MMSE becomes unstable when user 1 exceeds the rate of 0.6 packets/slot. In this region, the use of queue statistics becomes crucial. Note also that when queue statistics are used, the stability region of the true MMSE receiver is not much smaller than the clairvoyant receiver.

\section{CONCLUSIONS}

In this work, we propose a novel approach to PHY layer design in a multiple-access system-one that involves using queue statistics to implement an enhanced multi-user PHY layer. Specifically, we propose a linear MMSE front-end that explicitly uses the probability that a user has a packet to transmit in its design. Assuming a slotted ALOHA based MAC layer, we compared the stability region of the proposed receiver with other traditional receivers like the Matched Filter in the two user case and showed that the use of queue statistics becomes important, especially in near-far situations. Our approach is an example of cross layer design of the PHY and MAC layers and although our results are preliminary, they suggest that significant improvements in system design and performance are possible by such approaches. Cross-layer techniques present an alternative to traditional network design and the pros and cons of these techniques need to be investigated further.

\section{REFERENCES}

[1] L. Tong, Q. Zhao, and G. Mergen, "Multipacket reception in random access wireless networks: from signal processing to optimal medium access control," IEEE Communication Magazine, vol. 39, pp. 108112, Nov 2001. Special issue on Design Methodologies for Adaptive and Multimedia Networks.

[2] S. Adireddy and L. Tong, "Exploiting decentralized channel state information for random access," Submitted to IEEE Trans. Info. Theory, November 2002. http: / / acsp. ece. cornell.edu/pubJ.html.

[3] V. Naware and L. Tong, "Smart Antennas, Dumb Scheduling for Medium Access Control," in Proc. CISS 2003, (Baltimore, MD), March 2003.

[4] R. M. Loynes, "The stability of a queue with non-independant interarrival and service times," Proc. Cambridge Philos. Soc., vol. 58, pp. 497-520, 1962.

[5] B. Tsybakov and W. Mikhailov, "Ergodicity of slotted ALOHA systems," Probl. Inform. Transmission, vol. 15, p. 301?12, Oct.Dec. 1979.

[6] R.Rao and A.Ephremides, "On the stability of interacting queues in a multi-access system," IEEE Trans. Inform. Theory, vol. 34, pp. 918-930, September 1988.

[7] W.Szpankowski, "Stability conditions for some multiqueue distributed systems: buffered random access systems," Adv. Appl. Probab., vol. 26, pp. 498-515, 1994.

[8] V. Naware, G. Mergen, and L. Tong, "Stability and delay of finite user slotted ALOHA with multipacket reception," submitted to IEEE Trans. Inform. Theory, November 2003.

[9] W.Luo and A.Ephremides, "Stability of $N$ interacting queues in random-access Systems," IEEE Tran. Info. Theory, vol. 45, pp. 1579-1587, 1999. 